\title{
UTILIZAÇÃO DA EXTRAÇÃO EM FASE SÓLIDA (SPE) NA DETERMINAÇÃo DE HIDROCARBONETOS POLICÍCLICOS AROMÁTICOS EM MATRIZES AQUOSAS AMBIENTAIS
}

\author{
Rivelino M. Cavalcante, Nilton S. M. Filho, Rommel B. Viana, Isadora R. N. Oliveira e Ronaldo F. Nascimento* \\ Departamento de Química Analítica e Físico Química, Universidade Federal do Ceará, Av. Humberto Monte, s/n, \\ 60455-760 Fortaleza - CE, Brasil \\ Edilberto R. Silveira \\ Departamento de Química Orgânica e Inorgânica, Universidade Federal do Ceará, CP 12200, 60451-970 Fortaleza - CE, Brasil \\ George S. S. Freire \\ Departamento de Geologia, Universidade Federal do Ceará, Fortaleza - CE, Brasil \\ Recebido em 1/2/06; aceito em 7/7/06; publicado na web em 19/1/07
}

\begin{abstract}
UTILIZATION OF SOLID-PHASE EXTRACTION (SPE) FOR THE DETERMINATION OF POLYCYCLIC AROMATIC HYDROCARBONS IN ENVIRONMENTAL AQUEOUS MATRICES. Polycyclic aromatic hydrocarbons (PAHs) are a great environmental concern mainly because of their toxic, mutagenic and carcinogenic potential. This paper reports utilization of the solid-phase extraction (SPE) technique to determine PAHs in environmental aqueous matrices. The recovery from environmental aqueous matrices fortified with PAHs varied from 63.7 to $93.1 \%$ for atmospheric liquid precipitation, from 38.3 to $95.1 \%$ for superficial river water, and from 71.0 to $95.5 \%$ for marine water. No negative matrix effect was observed for the recovery of PAHs from atmospheric liquid precipitation and marine water, but was observed for superficial river water, particularly for PAHs possessing 5 and 6 aromatic rings.
\end{abstract}

Keywords: solid-phase extraction; water analysis, polycyclic aromatic hydrocarbons.

\section{INTRODUÇÃO}

Os hidrocarbonetos policíclicos aromáticos (HPAs) são de grande interesse ambiental, em virtude do seu potencial tóxico, mutagênico e carcinogênico ${ }^{1,2}$. Os HPAs que apresentam entre 4 e 6 anéis aromáticos são altamente mutagênicos e carcinogênicos, enquanto os de 2 a 3 anéis aromáticos, apesar de menos mutagênicos, são altamente tóxicos ${ }^{1-3}$.

Os HPAs são introduzidos no ambiente por fontes naturais e antrópicas ${ }^{4}$. As principais fontes naturais incluem a queima natural de florestas, as emissões vulcânicas e os afloramentos naturais de petróleo (processos petrogênicos) ${ }^{4}$. Alguns organismos, tais como bactérias, algas e fungos, também podem produzir naturalmente HPAs (processos biogênicos), todavia as fontes naturais são infinitamente insignificantes frente às fontes antrópicas ${ }^{5-7}$. As fontes antropogênicas de HPAs geralmente estão ligadas ao manuseio ou à combustão incompleta de matéria orgânica, especialmente combustíveis fósseis e seus derivados (processos pirogênicos). Em grandes centros urbanos, a principal fonte de HPAs para os corpos hídricos é através do "runoff" urbano, caracterizando-se como fonte pontual em grandes metrópoles ${ }^{8}$. Devido principalmente à baixa solubilidade, o que consequientemente favorece a alta hidrofobicidade ${ }^{3,9}$, os HPAs presentes na água apresentam grande afinidade pelas frações orgânicas particulada e dissolvida, podendo ser transportados a longas distâncias, alcançando inclusive áreas remotas ${ }^{5-7,9,10}$.

Para a determinação dos níveis de HPAs em água, geralmente se utilizam os métodos propostos pela US-EPA ou "Standard Methods"1. Para ambos é recomendada a extração líquido-líquido (ELL), seguida de pré-purificação ("clean-up") com sílica e determinação por cromatografia gasosa (CG) ou cromatografia líquida de alta eficiência (CLAE). A ELL é a técnica mais utilizada de extração de compostos orgânicos provenientes de matrizes aquosas,

*e-mail: ronaldo@ufc.br porém com a diminuição dos custos e praticidade no processo, a extração em fase sólida (SPE) está crescentemente sendo utilizada na quantificação de HPAs ${ }^{12-15}$.

A SPE, além de extrair, concentrar e pré-purificar os analitos, também utiliza pequenos volumes de solventes e pouca manipulação da amostra, o que corrobora para a redução no tempo da análi$\mathrm{se}^{16}$. Apesar da considerável eficiência da SPE na análise de compostos orgânicos, existe um número de fatores que influenciam na recuperação de $\operatorname{HPAs}^{12,14,17}$. Conseqüentemente, este artigo se propôs a investigar a utilização da SPE na determinação de HPAs em matrizes aquosas ambientais, bem como suas principais variantes, tais como o efeito da adição de co-solvente na amostra; razão do fluxo de percolação da amostra sobre os cartuchos de SPE; eluição dos HPAs nos cartuchos e o efeito matriz causado na eficiência de recuperação. As melhores condições encontradas, utilizando SPE, foram usadas nas análises de HPAs em matrizes ambientais e a eficiência de recuperação comparada com método padrão do "Standard Methods".

\section{PARTE EXPERIMENTAL}

\section{Material}

Foram utilizados em todos os experimentos, incluindo a limpeza de material, solventes grau cromatográfico bidestilados e água Milli-Q (sistema Millipore). Os padrões dos 16 HPAs foram obtidos da Sigma-Aldrich (USA), apresentando entre 99,5 e 99,9\% de pureza. Os HPAs estudados foram: Naftaleno (NAP); Acenaftileno (ACY); Acenafteno (ACE ); Fluoreno (FL); Fenantreno (PHEN); Antraceno (ANT); Fluoranteno (FLR); Pireno (PYR); Benzo(a)antraceno (BaA); Criseno (CHRY); Benzo(b)fluoranteno (BbF); Benzo(k)fluoranteno (BkF); Benzo(a)pireno (BaP); Indeno(1,2,3-cd)pireno (INP); Dibenzo(a,h)antraceno (DahA) e Benzo(g,h,i)perileno (BghiP). A sílica gel e o $\mathrm{Na}_{2} \mathrm{SO}_{4}$ anidro foram obtidos da Merck (USA) e Vetec, 
respectivamente. Foram utilizados cartuchos de SPE (Spe-ed ${ }^{\mathrm{TM}}, 0,5$ $\mathrm{g} / 6 \mathrm{~mL}$, marca Applied Separations) de Octadecyl $\left(\mathrm{C}_{18} / 14 \% \mathrm{C}\right)$, acoplado a um sistema de vácuo tipo "manifold".

\section{Procedimentos}

Oito HPAs (NAP, ACE, ANT, FLR, PYR, BaP, DahA e BghiP) foram utilizados na otimização da técnica de SPE. Os HPAs selecionados apresentam de 2 a 6 anéis aromáticos, representado assim a classe dos 16 HPAs prioritários pela US-EPA². Foi preparada uma solução estoque de $100 \mathrm{mg} / \mathrm{L}$ dos oito HPAs. Para cada estudo 100 $\mathrm{mL}$ (10 ng HPAs/mL) de amostras em duplicatas foram preparadas com as percentagens dos respectivos co-solventes (metanol ou acetona), nas percentagens de $10,20,30 \mathrm{ou} 40 \%$. O efeito da velocidade de fluxo sobre a recuperação dos HPAs foi estudado através dos fluxos de 5,10 e $15 \mathrm{~mL} / \mathrm{min}$. Foram avaliados como solventes de eluição dos HPAs nos cartuchos de SPE acetona, acetonitrila (ACN), metanol $(\mathrm{MeOH})$, acetona/tetraidrofurano (THF) $(1: 1, \mathrm{v} / \mathrm{v})$ e acetona/ diclorometano (DCM) (1:1, v/v) nos volumes de 2x1 e 4x1 mL.

$\mathrm{O}$ condicionamento dos cartuchos de SPE foi realizado pela passagem de $10 \mathrm{~mL}$ de água e, em seguida, $10 \mathrm{~mL}$ de água mais co-solvente (nas percentagens de 10, 20, 30 ou 40\%). As amostras foram então percoladas através dos cartuchos em fluxos de 5, 10 ou $15 \mathrm{~mL} / \mathrm{min}$. Após, serem lavados com $10 \mathrm{~mL}$ de água Milli-Q, os cartuchos foram centrifugados por $45 \mathrm{~min}$ a $6000 \mathrm{rpm}$ e, para garantir a ausência de água, mantidos por 10 min sob fluxo de nitrogênio ultrapuro. A eluição foi conduzida através da passagem do solvente orgânico com volumes de $2 \times 1$ ou $4 \times 1 \mathrm{~mL}$, a um fluxo entre 1 e $2 \mathrm{~mL} / \mathrm{min}$. Em seguida, as amostras tiveram seus volumes reduzidos para $0,5 \mathrm{~mL}$, utilizando-se fluxo de nitrogênio.

\section{Avaliação do efeito matriz}

$\mathrm{O}$ efeito matriz foi estudado utilizando-se as melhores condições encontradas para recuperação de HPAs por SPE. A extração de HPAs também foi realizada através da ELL ${ }^{11}$, para efeito de comparação. Para ambos foram utilizados $220 \mathrm{~mL}$ das matrizes ambientais (precipitação líquida atmosférica, água superficial de rio e água superficial marinha) em duplicatas. As amostras foram filtradas, o $\mathrm{pH}$ foi mantido neutro, fortificadas em seguida com concentrações variando entre 0,43 a $1,97 \mathrm{ng} / \mathrm{mL}$ dos 16 HPAs prioritários da US-EPA e mantidas sob agitação por $4 \mathrm{~h}$. Todas as matrizes ambientais usadas neste estudo foram analisadas em branco sem a adição de HPAs. A recuperação $(R)$ foi realizada utilizando a Equação.

$$
R=\frac{\text { massa do analito após análise }}{\text { massa do analito adicionada }} \times 100
$$

Para estudo do efeito matriz utilizando a SPE, após a fortificação, adicionou-se acetona (como co-solvente) às amostras ambientais, perfazendo $30 \%$ da solução de $220 \mathrm{~mL}$. Em seguida, as amostras foram percoladas através dos cartuchos sob fluxo entre 10 e $15 \mathrm{~mL} / \mathrm{min}$. Os cartuchos foram, então, lavados com 10 $\mathrm{mL}$ de água Milli-Q, centrifugados por $45 \mathrm{~min}$ a $6000 \mathrm{rpm}$, mantidos sob fluxo de nitrogênio ultrapuro por $10 \mathrm{~min}$ e, a seguir, eluídos com $2 \times 1 \mathrm{~mL}$ de acetona/THF $(1: 1, \mathrm{v} / \mathrm{v})$ a um fluxo de $1-2 \mathrm{~mL} / \mathrm{min}$. $\mathrm{O}$ volume do solvente foi reduzido para $500 \mu \mathrm{L}$ utilizando fluxo de nitrogênio e, em alguns casos, foi reduzido para $100 \mu \mathrm{L}$, no intuito de facilitar a detecção dos compostos de menor sensibilidade.

Na ELL foi utilizado o procedimento 6440 do "Standart Methods"11. Brevemente, $220 \mathrm{~mL}$ da matriz aquosa ambiental foram extraídos com $450 \mathrm{~mL}$ DCM, o volume reduzido para $1 \mathrm{~mL}$ e o DCM trocado por cicloexano. Foi preparada uma coluna cromatográfica ( $25 \mathrm{~cm}$ x $1 \mathrm{~cm}$ d.i.) contendo $10 \mathrm{~g}$ sílica gel e $\mathrm{Na}_{2} \mathrm{SO}_{4}$ (no topo) em DCM. Primeiramente, a coluna foi pré-eluída com 40 $\mathrm{mL}$ de pentano, adicionado o extrato em cicloexano e mais $25 \mathrm{~mL}$ de pentano eram passados através da coluna. Finalmente, um volume de $25 \mathrm{~mL}$ de DCM/pentano (4:6, v/v) foi passado através da coluna, recolhido, reduzido para $500 \mu \mathrm{L}$ e, em seguida, analisado.

\section{Análise por CG}

Foi utilizando cromatógrafo gasoso, modelo CG17A-Shimadzu, interfaciado com detector de ionização por chama (DIC), com uma coluna DB-5 J\&W Scientific (30 m x 0,25 mm d.i. x $0.25 \mu \mathrm{m}$ de filme) acoplada. A temperatura do injetor e detector era de 280 e 300 ${ }^{\circ} \mathrm{C}$, respectivamente. A temperatura de programação teve início a 85 ${ }^{\circ} \mathrm{C}$ permanecendo por $5 \mathrm{~min}$, aumentando para $120^{\circ} \mathrm{C}$ a uma taxa de $30{ }^{\circ} \mathrm{C} / \mathrm{min}$, em seguida para $230^{\circ} \mathrm{C}$ a uma taxa de $5^{\circ} \mathrm{C} / \mathrm{min}$, e, finalmente, para $305{ }^{\circ} \mathrm{C}$ a $3{ }^{\circ} \mathrm{C} / \mathrm{min}$. Foram injetados $2 \mu \mathrm{L}$ de amostra no modo "split" (1:20), utilizando hidrogênio como gás de arraste a um fluxo de 1,0 $\mathrm{mL} / \mathrm{min}$. Para melhorar a definição, principalmente dos últimos HPAs eluídos da coluna, foi utilizado um programa de pressão (início em 53 até $98 \mathrm{kpa}$ a uma taxa de $2 \mathrm{kpa} / \mathrm{min}$ ).

\section{RESULTADOS E DISCUSSÃO}

\section{Adição de co-solvente na amostra}

Em virtude da baixa solubilidade dos HPAs, a qual decresce com o aumento da massa molecular, a amostragem dos respectivos compostos pode ser prejudicada devido à adsorção nas paredes dos recipientes utilizados para tal procedimento ${ }^{14}$. Portanto, a adição de solventes orgânicos ( $\mathrm{MeOH}, \mathrm{ACN}$ ou 2-propanol), chamados também de co-solventes ou modificantes orgânicos, é um recurso para aumentar a solubilidade dos HPAs, diminuindo a adsorção nas paredes dos frascos de amostragem ${ }^{14,15,18}$. Entretanto, a concentração do co-solvente é um parâmetro crítico, o qual deve ser avaliado precisamente, levando-se em consideração que se adicionado em baixa concentração pode não dissolver completamente os compostos de maiores massas moleculares, mas que se adicionado em alta concentração dificulta a adsorção dos compostos com menores massas moleculares ${ }^{12,14,19}$. Desta forma, para cada tipo de adsorvente e co-solvente este parâmetro deve ser otimizado.

Como pode ser observado na Figura 1, utilizando-se $20 \%$ de metanol como co-solvente a recuperação do NAP e ACE foi acima de $90 \%$. O NAP e ACE também apresentaram recuperações satisfatórias (70 a 80\%) para as outras concentrações de metanol estudadas. Diferentemente, isto não foi observado para os demais HPAs estudados em outras concentrações de metanol como cosolvente (Figura 1a). Para ANT, FLR e PYR a recuperação foi entre 30 a $60 \%$ para as concentrações de 10 a $40 \%$ de co-solvente (Figura 1a). Menor eficiência na recuperação, variando de não detectado (nd) a 30\%, foi observada para os compostos de maiores massas moleculares, como BaP, DahA e BghiP. Utilizando-se a acetona como co-solvente, melhores resultados foram obtidos, comparados aos do metanol (Figuras 1a e 1b). Como pode ser verificado na Figura 1b, utilizando a acetona na concentração de 30 e 40\%, a recuperação da maioria dos HPAs foi melhor quando comparada à concentração de 10 e 20\% (Figura 1b). A recuperação dos HPAs variou de 74,4 a $95,8 \%$, (30\% acetona) e de 51,6 a $108,5 \%(40 \%$ acetona). $\mathrm{O}$ metanol $\left(\mathrm{e}^{\circ}=0,73\right)$ apresenta valor eluotrópico quase duas vezes maior que a acetona $\left(\mathrm{e}^{\circ}=0,43\right)$, assim para consideráveis volumes ocorre a diminuição na adsorção pelos sítios dos adsorventes. Nkedl-Kizza e colaboradore ${ }^{20}$ estudaram a influência da mistura metanol/água e acetona/água na adsorção de HPAs e 
obtiveram coeficientes de adsorções maiores para a acetona frente ao metanol.

A utilização de acetona 30 ou $40 \%$ como co-solvente apresentou recuperação semelhante aos estudos de Falcon e colaboradores $^{19}$, utilizando ACN 30\% (recuperação de 70-105\%) e Urbe e Ruana $^{18}$ utilizando isopropanol $10 \%$ (recuperação de 58-105\%). A acetona foi superior, principalmente na recuperação dos HPAs de 2 e 3 anéis aromáticos, quando comparada com os resultados de Martinez e colaboradores ${ }^{15}$, utilizando metanol $10 \%$ (recuperação de $35-113 \%$ ) e Kiss e colaboradores ${ }^{12}$, utilizando 2-propanol $25 \%$ (recuperação de 10-98\%). Em virtude da diferença insignificante na recuperação de HPAs utilizando acetona 30 ou $40 \%$ como cosolvente e, conseqüentemente, menor utilização de solvente, o que diminui custos e contribui para a redução do uso de compostos orgânicos, adotou-se no estudo a acetona $30 \%$.
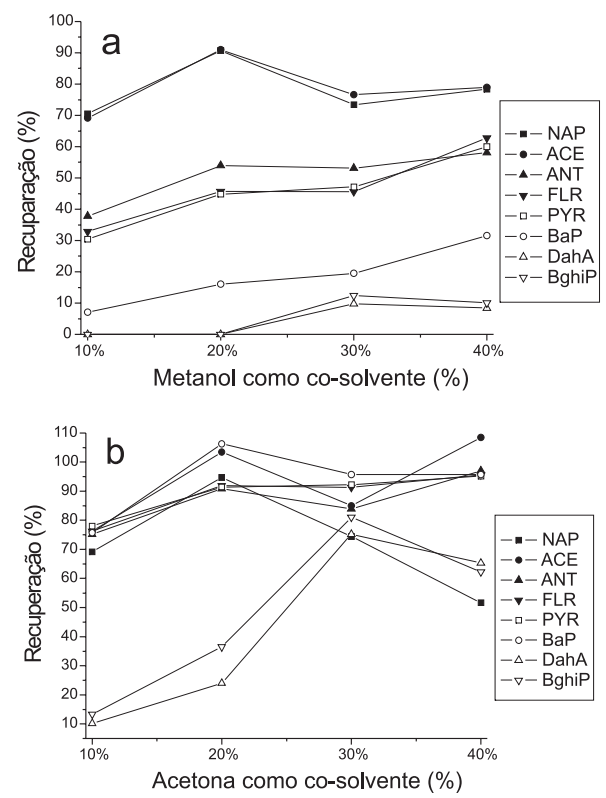

Figura 1. Efeito na recuperação de HPAs utilizando (a) metanol e (b) acetona como co-solventes em $100 \mathrm{~mL}$ de amostra e $2 \mathrm{~mL}$ de acetona/THF como eluente

\section{Efeito da razão de fluxo na percolação de HPAs nos cartuchos de SPE}

Diversos valores para o fluxo de percolação através dos cartuchos de SPE são utilizados. Muitos trabalhos utilizaram 2 a $25 \mathrm{~mL} /$ $\min ^{12,15,19,21}$, fabricantes recomendam uma faixa de trabalho entre 2 a $50 \mathrm{~mL} / \mathrm{min}^{22,23}$. O fluxo de percolação através dos cartuchos é um fator governante, uma vez que diminui o tempo de preparo das amostras e, conseqüentemente, de análise. Em contrapartida, altos fluxos podem diminuir o tempo de equilíbrio necessário na adsorção, levando à diminuição na eficiência da recuperação dos compostos estudados ${ }^{12,14}$.

Neste estudo, a recuperação de HPAs variou de 73,2 a 94,2\% ( $5 \mathrm{~mL} / \mathrm{min}), 71,3$ a $100,1 \%(10 \mathrm{~mL} / \mathrm{min})$ e 69,1 a $99,1 \%(15 \mathrm{~mL} /$ min). Na Figura 2 pode ser verificado um decréscimo na recuperação de NAP, ACE, ANT e DahA com o aumento do fluxo de percolação. Por outro lado, a recuperação é maior para FLR, PYR, $\mathrm{BaP}$ e BghiP quando o fluxo é aumentado para 10 e $15 \mathrm{~mL} / \mathrm{min}$. Em virtude da diferença dos fluxos estudados não apresentarem diferença significativa, da necessidade de menor tempo para análise e dificuldade em manter o fluxo homogêneo para todos os cartuchos de SPE, foi adotada a velocidade de fluxo entre 10 e $15 \mathrm{~mL} /$ min na percolação das amostras.

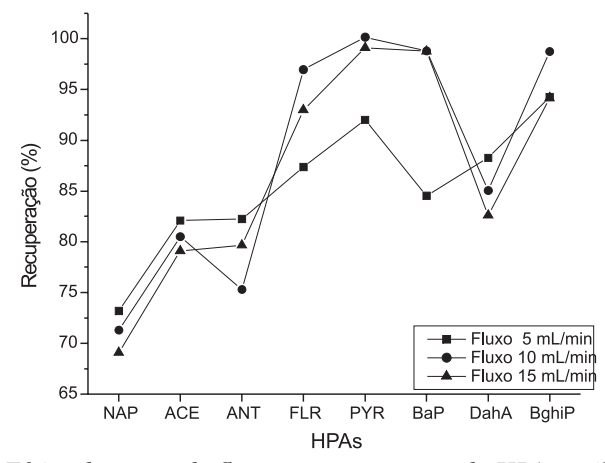

Figura 2. Efeito da razão de fluxo na recuperação de HPAs, utilizando 100 $\mathrm{mL}$ de amostra em 30\% de acetona como co-solvente e $2 \mathrm{~mL}$ de acetona/THF como eluente

\section{Eluição dos HPAs nos cartuchos de SPE}

Utilizando o volume de $4 \times 1 \mathrm{~mL}$ dos respectivos solventes de eluição, pode observar-se na Figura 3b, que somente metanol, acetona/THF e acetona/DCM apresentaram recuperação satisfatória para a maioria dos HPAs estudados. Por outro lado, utilizando o volume de 2x1 mL (Figura 3a), somente as misturas de acetona/ THF e acetona/DCM apresentaram recuperação satisfatória. O teste ANOVA "one way" utilizando o software STATISTIC $6^{\mathrm{TM}}$ não apresentou diferença significante (nível de $\mathrm{P}<0,001$ ) entre 2 e 4 $\mathrm{mL}$ de acetona/THF e acetona/DCM na eficiência de recuperação dos HPAs estudados. A mistura de $2 \mathrm{~mL}$ de acetona/THF foi selecionada para o restante do estudo como solvente de eluição, principalmente em virtude do protocolo de Kyoto, o qual sugere a diminuição do uso de compostos organo-clorados, bem como a opção de se utilizar a cromatografia líquida.
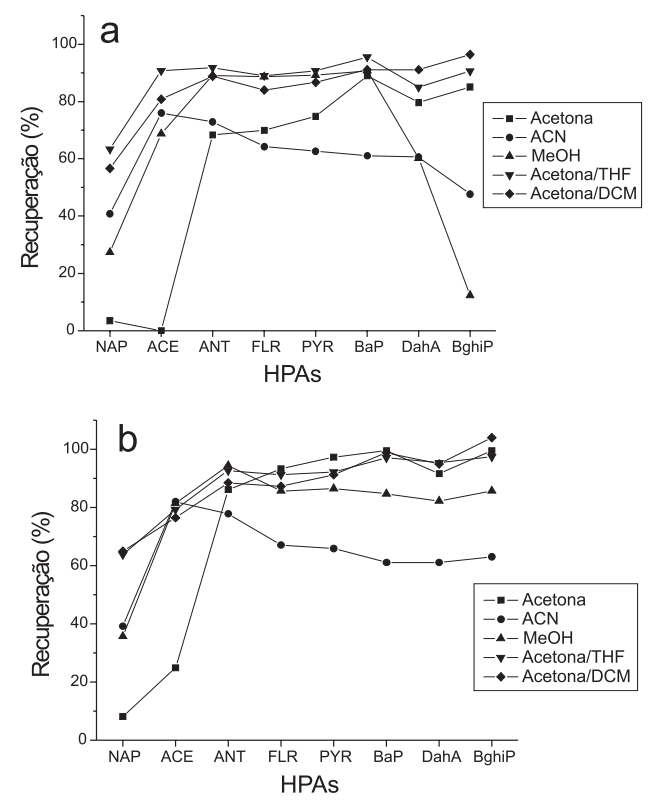

Figura 3. Efeito na recuperação de HPAs utilizando (a) $2 \mathrm{~mL}$ e (b) $4 \mathrm{~mL}$ como solventes de eluição em amostra em $30 \%$ de acetona como co-solvente

\section{Efeito matriz na recuperação de HPAs proveniente de} matrizes ambientais

As recuperações em matrizes ambientais fortificadas com HPAs podem ser verificadas na Figura 4. A recuperação variou de 63,7 a 93,1\% para a precipitação líquida atmosférica, de 38,3 a 95,1\% para 
Tabela 1. Média e desvio padrão da recuperação (\%) de HPAs utilizando SPE e ELL na precipitação liquida atmosférica, água superficial de rio e água superficial marinha

\begin{tabular}{|c|c|c|c|c|c|c|}
\hline \multirow[t]{2}{*}{ HPAs } & \multicolumn{2}{|c|}{ Precipitação líquida atmosférica } & \multicolumn{2}{|c|}{ Água de rio } & \multicolumn{2}{|c|}{ Água marinha } \\
\hline & SPE $(\%)$ & ELL $(\%)$ & SPE $(\%)$ & ELL (\%) & $\operatorname{SPE}(\%)$ & ELL $(\%)$ \\
\hline NAP & $63,7 \pm 7,4$ & $60,0 \pm 8,2$ & $78,0 \pm 7,0$ & $34,2 \pm 8,5$ & $72,5 \pm 5,3$ & $54,9 \pm 21,1$ \\
\hline $\mathrm{ACY}+\mathrm{ACE}$ & $79,2 \pm 2,5$ & $79,7 \pm 12,4$ & $76,0 \pm 12,3$ & $51,4 \pm 4,7$ & $82,9 \pm 4,8$ & $70,5 \pm 13,8$ \\
\hline FL & $88,8 \pm 9,7$ & $81,3 \pm 20,1$ & $85,4 \pm 2,9$ & $76,3 \pm 9,2$ & $93,0 \pm 6,1$ & $99,0 \pm 16,4$ \\
\hline PHEN & $91,2 \pm 8,3$ & $75,3 \pm 6,4$ & $95,1 \pm 8,6$ & $80,9 \pm 8,2$ & $94,8 \pm 10,3$ & $88,2 \pm 23,3$ \\
\hline ANT & $93,1 \pm 9,5$ & $80,9 \pm 3,8$ & $89,4 \pm 5,8$ & $83,7 \pm 16,9$ & $95,5 \pm 17,1$ & $79,1 \pm 24,1$ \\
\hline FLR & $86,0 \pm 8,1$ & $89,0 \pm 11,3$ & $77,0 \pm 9,1$ & $63,6 \pm 19,2$ & $83,9 \pm 7,9$ & $90,5 \pm 5,6$ \\
\hline PYR & $77,9 \pm 10,6$ & $83,0 \pm 13,4$ & $69,4 \pm 8,6$ & $76,1 \pm 4,1$ & $79,7 \pm 7,1$ & $90,4 \pm 7,6$ \\
\hline $\mathrm{BaA}$ & $72,7 \pm 18,1$ & $83,8 \pm 21,1$ & $65,5 \pm 9,0$ & $94,3 \pm 12,9$ & $89,4 \pm 13,0$ & $93,6 \pm 12,7$ \\
\hline CHRY & $75,0 \pm 22,4$ & $82,8 \pm 22,2$ & $76,0 \pm 8,3$ & $80,6 \pm 8,5$ & $82,6 \pm 6,5$ & $78,3 \pm 25,5$ \\
\hline $\mathrm{BbF}$ & $71,1 \pm 22,7$ & $77,5 \pm 20,4$ & $51,5 \pm 7,8$ & $85,5 \pm 15,1$ & $73,1 \pm 12,4$ & $84,4 \pm 6,7$ \\
\hline $\mathrm{BkF}$ & $81,0 \pm 16,3$ & $68,1 \pm 20,6$ & $58,1 \pm 14,0$ & $69,2 \pm 15,3$ & $77,5 \pm 17,5$ & $80,6 \pm 7,9$ \\
\hline $\mathrm{BaP}$ & $77,3 \pm 16,6$ & $69,0 \pm 16,8$ & $52,1 \pm 13,6$ & $71,6 \pm 9,0$ & $71,0 \pm 11,1$ & $62,1 \pm 10,3$ \\
\hline IND & $70,1 \pm 13,5$ & $76,0 \pm 22,0$ & $38,3 \pm 16,4$ & $78,2 \pm 20,5$ & $74,5 \pm 15,7$ & $80,0 \pm 11,7$ \\
\hline DahA & $76,5 \pm 7,1$ & $84,5 \pm 25,4$ & $42,0 \pm 16,7$ & $95,0 \pm 15,3$ & $85,1 \pm 14,6$ & $97,2 \pm 12,8$ \\
\hline BghiP & $74,4 \pm 10,3$ & $76,0 \pm 19,1$ & $50,7 \pm 12,8$ & $89,1 \pm 17,4$ & $80,2 \pm 15,6$ & $86,5 \pm 13,1$ \\
\hline
\end{tabular}

água superficial de rio, de 71,0 a 95,5\% para água superficial marinha e de 68,4 a 100,4\% para água Milli-Q. O efeito matriz observado frente à recuperação de HPAs em água Milli-Q, só foi observado para água superficial de rio e, principalmente, para os HPAs de 5 e 6 anéis. $\mathrm{O}$ efeito negativo é em virtude da existência de altas concentrações de ácidos húmicos nesta matriz, os quais adsorvem os HPAs, evitando a retenção pelos sítios dos adsorventes ${ }^{10,21}$.

Urbe e Ruana ${ }^{18}$ não observaram efeito negativo na recuperação de HPAs provenientes de amostras de água de rio fortificadas, porém, Martinez e colaboradores ${ }^{15}$ encontraram comportamento semelhante ao observado neste estudo, ou seja, a diminuição para uma faixa entre 40 a $60 \%$ na recuperação dos HPAs de 5 e 6 anéis. Diferentemente da água de rio, a precipitação líquida atmosférica e a água marinha apresentam ausência ou baixíssima concentração de ácidos húmicos, porém especialmente para a água marinha, outros tipos de constituintes podem apresentar efeito matriz negativo. Segundo Means ${ }^{24}$, a salinidade pode diminuir a recuperação de HPAs quando se utiliza a SPE. Urbe e Ruana ${ }^{18}$, bem como Filipkowska e colaboradores $^{25}$, não relatam efeito negativo promovido por águas marinhas fortificadas com HPAs, o que condiz com os presentes resultados (Figura 4).

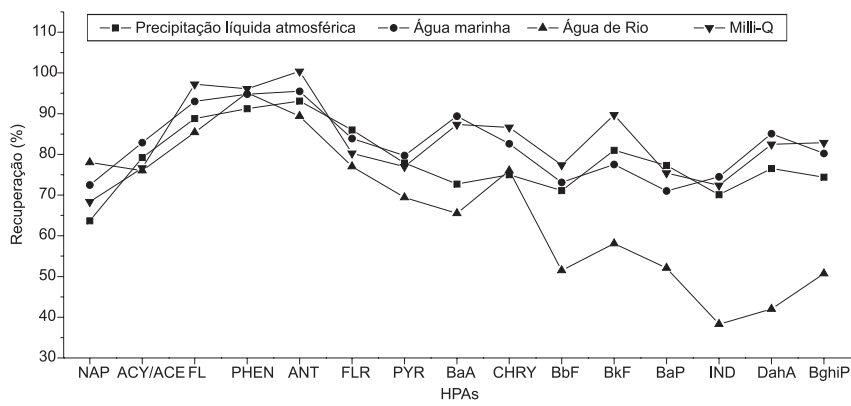

Figura 4. Recuperação de HPAs utilizando SPE

\section{Comparação entre ELL e SPE}

Para efeito de comparação, as mesmas matrizes ambientais fortificadas com HPAs foram também analisadas através da técnica ELL do "Standard Methods" ". A recuperação dos HPAs por ambas as técnicas está sumarizada na Tabela 1. No geral, utilizan- do-se a técnica de SPE, os desvios padrões foram menores ou ficaram próximos dos observados com a técnica de ELL.

Utilizando o teste $\mathrm{F}$ unilateral, o qual verifica se a precisão da técnica de SPE é significativamente menor que a do método considerado padrão (técnica ELL do "Standard Methods"), nós verificamos que para os HPAs de maiores massas moleculares (BaA, BbF, IND, DahA e BghiP) na matriz de água superficial de rio, as variâncias da técnica de SPE são significativamente maiores frente à ELL, em nível de 5\%. Desta forma, a técnica de SPE não é precisa na determinação de HPAs de maiores massas moleculares provenientes da matriz de água superficial de rio.

Em termos de análises rotineiras, a técnica de SPE é relativamente simples, eficiente, reprodutível e considerando-se a utilização de um sistema de vácuo "manifold", é mais rápida e menos exaustiva quando comparada à ELL. Além do mais, consome um volume de solvente menor, sendo importante salientar a utilização de um grande volume de DCM (carcinogênico ${ }^{1}$ ) consumido na ELL.

\section{CONCLUSÃO}

A otimização da técnica de SPE para análise de HPAs em matrizes ambientais apresentou melhor recuperação quando se utilizou acetona $30 \%$ como co-solvente, fluxo de percolação entre 10 e 15 $\mathrm{mL} / \mathrm{min}$ e $2 \mathrm{~mL}$ de acetona/THF como mistura de eluição. Não foi observado efeito matriz frente à recuperação de HPAs em amostras de precipitação líquida atmosférica e água superficial marinha, porém, foi observado um decréscimo na recuperação dos HPAs de maiores massas moleculares em água superficial de rio. A técnica de SPE apresentou satisfatória eficiência na recuperação e reprodutibilidade frente à comparação com o método padrão, além de reduzido consumo de solvente e facilidade na etapa do preparo da amostra.

\section{REFERÊNCIAS}

1. http://www.iarc.fr, acessada em Novembro 2005.

2. http://www.epa.gov/iris, acessada em Novembro 2005.

3. Pereira Netto, A. D.; Moreira, J. C.; Dias, A. E. X. O.; Arbilla, G.; Ferreira, L. F. V.; Oliveira, A. S.; Barek, J. Quim. Nova 2000, 23, 765.

4. Hinchee, R. E.; Alleman, B. C.; Hoeppel, R. E.; Miller, R. N.; CRC Press 1994, p. 105-152.

5. Reddy, C. M.; Quinn, J. G.; Marine Poll. Bull. 1999, 38, 126.

6. Soclo, H. H.; Garrigues, P. H.; Ewald, M.; Marine Poll. Bull. 2000, 40, 387. 
7. Tam, N. F. Y.; Ke, L.; Wang, X. H.; Wong, Y. S.; Environ. Pollut. 2001, $114,255$.

8. Walker, W. J.; McNutt, R. P.; Maslanka, C. K.; Chemosphere 1999, 38, 363.

9. Lopes, W. A.; de Andrade, J. B. Quim. Nova 1996, 19, 497.

10. Vasconcellos, P. C.; Artaxo, P. E.; Ciccioli, P.; Cecinato, A.; Brancaleoni, E.; Frattoni, M.; Quim. Nova 1998, 21, 385.

11. Standard methods for the examination of water and wastewater. Individual organic compounds- Part 6000, PAH 6440, 20 th ed. (CD), 1998

12. Kiss, G.; Varga-Puchony, Z.; Labia, J.; J. Chromatogr., A 1996, 725, 261.

13. Kiss, G.; Gelencser, A.; Krivacsy, Z.; Hlavay, Z.; J. Chromatogr., A 1997, $774,349$.

14. Grynkiewicz, M.; Polkowska, \{.; Namie[nik, J.; Atmos. Environ. 2002, 36, 361.

14. Marcé, R.M.; Borrul, F.; J. Chromatogr., A 2000, 885, 273.

15. Martinez, E.; Gros, M.; Lacorte, S.;Barceló, D.; J. Chromatogr., A 2004, 1047,181
16. Aquino Neto, F. R.; Nunes, D. S. S.; Cromatografia-princípios básicos e técnicas afins, $1^{\text {a }}$ ed., Ed. Interciência: Rio de Janeiro, 2003, p. 25-30.

17. Lanças, F.; Extração em Fase Sólida (SPE), $4^{\mathrm{a}}$ ed., Ed. RiMa: São Carlos, 2004

18. Urbe, I.; Ruana, J.; J. Chromatogr., A 1997, 778, 337.

19. Falcon, M. S. G.; Lamela, M. P.; Gandara, J. S.; J. Agric. Food Chem. 2004, $52,6897$.

20. Nkedl-Kizza, P.; Rao, P. S. C.; Hornsby, A. G.; Environ. Sci. Technol. 1985 $19,975$.

21. Li, N.; Lee, H. K.; J. Chromatogr., A 2001, 921, 255.

22. Waters Sep-Pak DNPH-Silica cartridge; Care and use Manual, Waters Corporation: Milford, MA, 1994.

23. Applied Separations- Spe-ed; Instructions Manual, 2001

24. Means, J. C.; Mar. Chem. 1995, 51, 3.

25. Filipkowska, A.; Lubecki, L.; Kowalewska, G.; Anal. Chim. Acta 2005, 547, 243. 